\title{
La malattia policistica renale autosomica dominante dell'adulto: tributo alla sua identità
}

\section{S. Campo}

MMG, Responsabile dell'Area Urologica della Società Italiana di Medicina Generale

The Autosomal dominant POLYCySTIC Disease: TRIBUTE To ITS ESSENTIAL NATURE

Abstract. The autosomal dominant polycystic kidney disease (ADPKD) is one of the most common genetic diseases, with an incidence of 1:1000, and is the leading genetic cause of adult kidney failure. They can distinguish two types, type I, caused by mutations in the PKD1 gene, and type II, caused by mutations of the PKD2 gene. The disease has an onset between 40 and 50 years, but may occur sooner, and has a systemic footprint. The diagnosis is often accidental, in the course of investigations motivated by other clinical questions, or promoted by the presence of clinical signs related. In diagnostic procedures, the ADPKD must be distinguished from simple renal cysts, single or multiple, partially affecting the kidneys that usually do not present a critic evolution. It is possible that they can be misdiagnosed in clinical conditions involving the presence of renal cysts, with the risk of underestimating the clinical relevance of the ADPKD. To test this hypothesis, we conducted an epidemiological study in the setting of italian General Practice which showed a prevalence (1.9\%o) almost four times higher than that recorded (0.5\%o) before the information provided about the characteristics clinical and diagnostic of the ADPKD. The ratio M:F was 1.65. It is desirable the commitment of General Practice, in conjunction with the Patients' Associations and the nephrologic Scientific Societies, in order to contribute to an optimal clinical management of the condition, aimed to ensure the wellbeing of the patient.

Key Words. ADPKD, Autosomal dominant polycystic kidney disease, General Practice, Renal failure, Dialysis

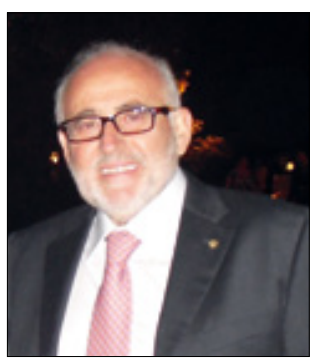

Salvatore Campo

\section{Introduzione}

La malattia policistica renale autosomica dominante dell'adulto (Autosomal dominant polycystic kidney disease $\mathrm{ADPKD}$ ) è una delle malattie genetiche più comuni, con un'incidenza di 1 su 1000 , ed è la principale causa genetica di insufficienza renale dell'adulto. Nel mondo, è la causa di terapia sostitutiva emodialitica nel $5-10 \%$ dei pazienti. È una malattia geneticamente eterogenea e se ne distinguono due tipi; il tipo I, causato da mutazioni del gene PKD1 che codifica per la policistina-1, è la forma più diffusa e aggressiva, responsabile di maggiore mortalità prematura e colpisce soggetti di età più giovane e il tipo II, causato da mutazioni del gene PKD2 che codifica per la policistina-2, che rappresenta il $10-15 \%$ dei casi, ad evoluzione più lenta.

Il gene PKD1, mutato in circa l'85\% dei pazienti, si trova sul cromosoma 16. Il gene PKD2, localizzato sul cromosoma 4 , risulta mutato nel rimanente $15 \%$ circa dei pazienti. L'ADPKD presenta penetranza completa, interessando tutti i 
soggetti che hanno ereditato la mutazione genetica, ed espressività variabile, in quanto l'epoca e i modi di insorgenza e di sviluppo delle cisti può essere variabile; questo comporta che, nell'attività clinica, bisogna sottoporre i congiunti di primo grado di un paziente affetto da ADPKD a uno screening diagnostico. E stata descritta una possibile condizione di eterozigosi per le mutazioni PKD1 e PKD2 (1-5). La malattia ha un esordio tra i 40 e i 50 anni, ma può manifestarsi anche prima, e ha un'impronta sistemica perché, oltre al rene, altri organi possono essere interessati dalle cisti (fegato, pancreas e milza) e, inoltre, è possibile un interessamento del sistema cardiovascolare relativamente alla presenza di ipertensione, di aneurismi cerebrali e coronarici, di dilatazioni dell'arco aortico e di valvulopatie cardiache. La malattia è anche associata, rispetto alla popolazione generale, a una maggiore prevalenza di diverticoli del colon e di ernie inguinali (1-4). La caratteristica principale della malattia consiste nel formarsi di cisti in entrambi i reni che progressivamente aumentano in numero e dimensioni fino a causare la perdita critica della funzionalità renale nella metà circa dei pazienti.

\section{La clinica}

Essendo la malattia, soprattutto all'esordio, frequentemente asintomatica, la diagnosi è spesso casuale, nel corso di indagini motivate da altri quesiti clinici; talvolta presenta segni clinici correlati (sensazione d'ingombro, palpazione di una massa in sede lombare, dolori lombari, micro-macro-ematuria, riduzione della clearance della creatinina ecc.) che portano alle indagini e alla diagnosi. L'ipertensione arteriosa è frequente e ne può essere una condizione di esordio clinico. Ne possono essere complicanze la nefrolitiasi, le infezioni urinarie e, anche se rara, la rottura delle cisti nelle vie urinarie (ematuria) o in sede retroperitoneale (dolore, febbre ecc.). La diagnosi strumentale è ecografica e, nei casi sospetti, con TAC. La diagnosi di certezza è genetica ma bisogna tener conto dei suoi costi e del fatto che riesce a dimostrare mutazioni certe in circa il $41-63 \%$ dei casi e mutazioni certe e verosimilmente associate a malattia nel $78-89 \%$ dei casi $(6,7)$. In Italia, è possibile eseguire la diagnosi genetica solo presso la Fondazione San Raffaele di Milano dove le UO di Nefrologia possono trasmettere un campione di sangue del paziente previo accordo. Nelle procedure diagnostiche, l'ADPKD deve essere distinta dalle cisti renali semplici, uniche o multiple, abbastanza comuni nell'adulto, che interessano parzialmente i reni e che generalmente non presentano un'evoluzione critica. Nella Tabella I sono elencate le condizioni con presenza di cisti renali, con codifica ICD-IX, e tra le quali il MMG deve saper scegliere nel codificare la condizione clinica del paziente nel software operativo di studio.

Alcune di queste condizioni hanno denominazioni che per assonanza potrebbero essere considerate dei sinonimi e se non viene effettuata una valutazione diagnostica attenta è possibile che le denominazioni possano essere usate indifferentemente. Considerato che nell'ordinaria attività clinica non è semplice procedere alla diagnosi genetica per la conferma diagnostica, è necessario ricorrere a criteri clinici e strumentali. Tra i criteri clinici, sono indicativi per ADPKD: insorgenza tra i 40 e i 50 anni o anche prima, evoluzione della malattia e familiarità. Nel 1994, Revine et al hanno definito le

TABELLA I - CONDIZIONI CHE COMPORTANO LA PRESENZA DI CISTI RENALI

\subsection{CISTI RENALI ACQUISITA; CISTI RENE}

753.1 MALATTIA CISTICA RENE

753.11 CISTI RENALE CONGENITA, SINGOLA

753.12 RENE POLICISTICO

753.13 RENE POLICISTICO AUTOSOMICO DOMINANTE (ADPKD)

753.14 RENE POLICISTICO AUTOSOMICO RECESSIVO

753.17 RENE CON MIDOLLARE A SPUGNA

753.19 RENE MULTICISTICO 
TABELLA II - CARATTERISTICHE ECOGRAFICHE CHE POS SONO SUGGERIRE LA DIAGNOSI DI ADPKD (9)

\begin{tabular}{|l|l|}
\hline $15-39$ anni & 3 o più cisti uni o bilaterali \\
\hline $40-59$ anni & 2 o più cisti in ciascun rene \\
\hline$\geq 60$ anni & 4 o più cisti in ciascun rene \\
\hline
\end{tabular}

caratteristiche ecografiche utili a diagnosticare il PKD1 e consistenti nella presenza di almeno due cisti (uni o bilaterali) tra i 15 e i 29 anni e la presenza di almeno due cisti in ciascun rene tra i 30 e i 59 anni (8). Uno studio di Pei et al, pubblicato nel 2009 sul Journal of the American Society of Nephrology, ha dato un consistente contributo per una più accurata diagnosi. Gli Autori hanno esaminato 577 soggetti da 58 famiglie a rischio di PKD1 e 371 da 39 famiglie a rischio di PKD2; li hanno sottoposti a indagini ecografiche e genetiche e dalla valutazione dei dati di sensibilità e specificità hanno estrapolato dei criteri diagnostici ecografici predittivi, in assenza di uno studio genetico, per ADPKD (9). Nella Tabella II sono rappresentate le caratteristiche ecografiche che possono suggerire la diagnosi di ADPKD.

Per altro verso, fra i 30 e 39 anni, l'assenza di riscontro ecografico di cisti può far escludere la presenza di malattia e, sopra i 40 anni, il riscontro ecografico di nessuna cisti o di una sola cisti renale ha un valore predittivo negativo del 100\%, con false-negative rate dello 0.7\% (9). Pertanto, complessivamente, la diagnosi può derivare dai seguenti criteri clinico-strumentali:

$\checkmark$ Insorgenza 40-50 anni o meno

$\checkmark$ Caratteristiche ecografiche delle cisti (Tab. II)

$\checkmark$ Evoluzione della malattia

$\checkmark$ Familiarità

$\checkmark$ Esame genetico positivo

Attualmente, non sono disponibili terapie capaci di arrestare o rallentare l'evoluzione della ADPKD; è possibile, invece, controllare le comorbidità o le complicanze per evitare che costituiscano componenti peggiorative per l'evoluzione della malattia. In tal senso, la sorveglianza clinica deve essere indirizzata, oltre a un monitoraggio della funzione renale, al controllo ottimale dell'ipertensione arteriosa e di altri fattori di rischio cardiovascolari che possono contribuire a rendere più severo il danno renale, alla confer- ma o meno di condizioni associate alla presenza di cisti, alla prevenzione delle infezioni e della formazione di calcoli ecc. La dimostrazione che la Policistina-1 si complessa con la tuberina e agisce come un inibitore endogeno dell'attività del mammalian Target of Rapamycin (mTOR) ha destato molto interesse ai fini terapeutici. L'uso di un inibitore di mTOR (Rapamicina o il derivato Everolimus), in pazienti affetti da ADPKD, inibendo l'mTOR, determinerebbe l'arresto del ciclo cellulare e quindi l'apoptosi (10). I recenti discordanti risultati di alcuni studi sull'uso degli inibitori dell'm-TOR nell'uomo possono generare interrogativi circa la loro posologia e il momento ottimale del trattamento e confusione sui reali benefici clinici della terapia (11). È il primo vero tentativo della ricerca scientifica di porre freno all'evoluzione della malattia e, anche se i primi risultati non sono entusiasmanti, può essere l'inizio di un percorso che potrebbe portare i pazienti affetti a guardare il futuro con maggiore fiducia.

\section{Perché l'attenzione della Medicina Generale italiana per l'ADPKD?}

Alcune motivazioni, epidemiologiche e cliniche, hanno indotto la Medicina Generale italiana ad impegnarsi per una migliore conoscenza dell'ADPKD:

1. I dati epidemiologici italiani disponibili sono parziali in quanto riguardanti campioni non significativi dell'intera popolazione.

2. Il non corretto inquadramento diagnostico dell'ADPKD può indurre a sottovalutare il rilievo clinico della malattia, per il rischio di insufficienza renale e come condizione ad alto rischio cardiovascolare.

3. La corretta diagnosi ha significato clinico per il paziente e, essendo una malattia autosomica dominante, valutativo-diagnostico per i familiari di I grado; pertanto la presa in carico riguarda sia il paziente sia la relativa famiglia. Per questo abbiamo voluto iniziare un percorso che possa portare la Medicina Generale a conoscere meglio la malattia per gestire al meglio le persone che ne sono affette. L'Associazione Italiana Rene Policistico (AIRP) ha voluto coinvolgere la Società Italiana di Medicina Generale (SIMG) nella tappa del suo Road Show 20102012 che il 31 marzo 2012 ha interessato Paler- 
AIRP

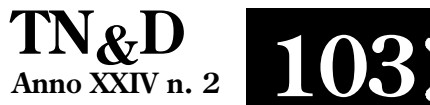

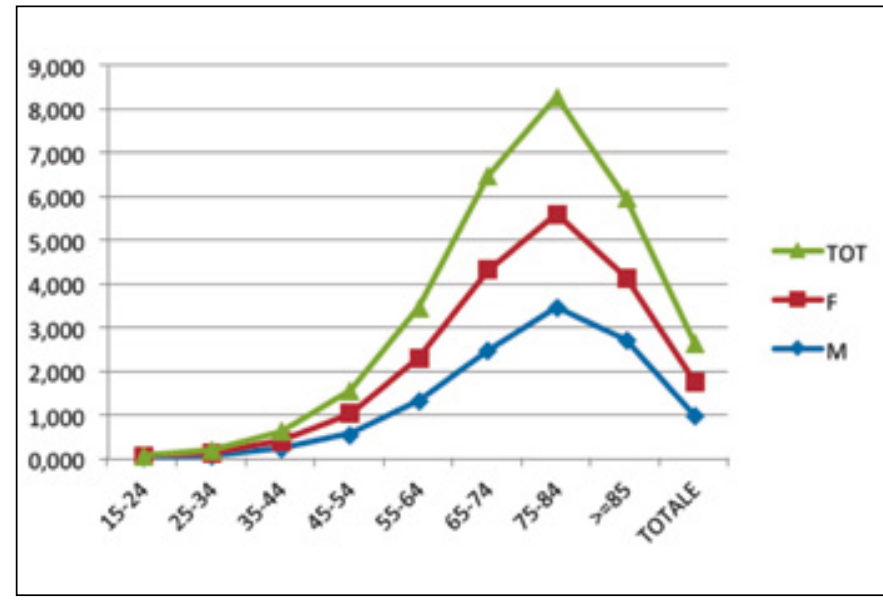

Grafico 1 - 593.2 Cisti renale acquisita; cisti rene.

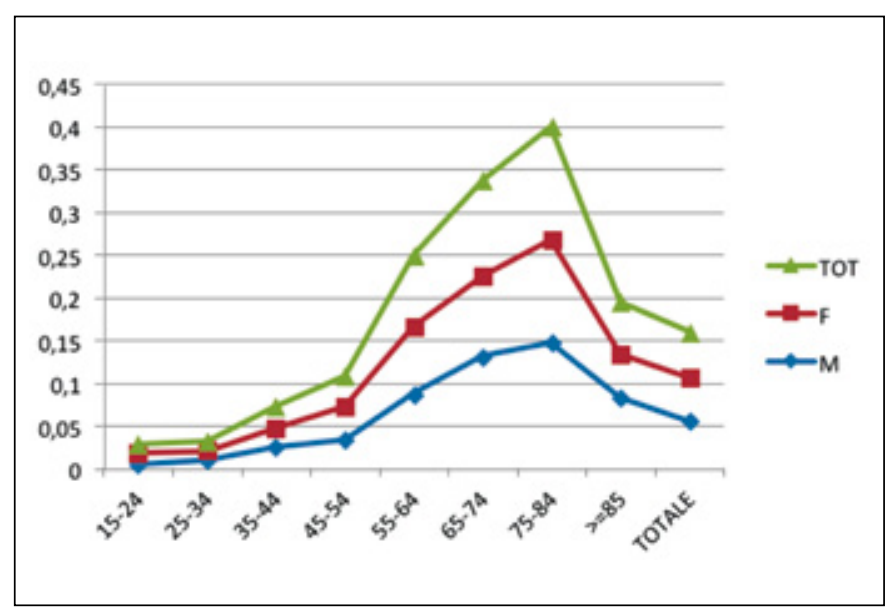

Grafico 3 - 753.11 Cisti renale congenita, singola

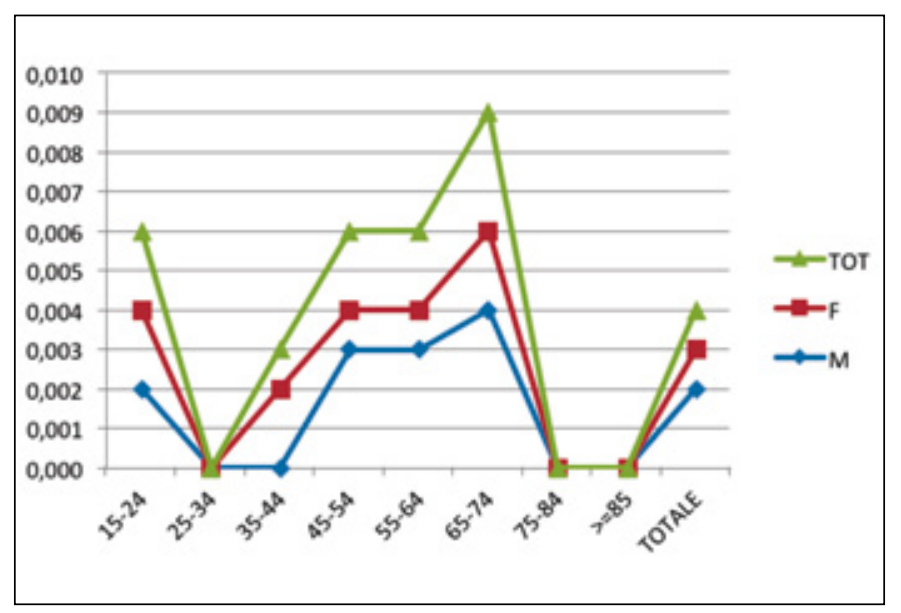

Grafico 5 - 753.13 Rene Policistico Autosomico Dominante (ADPKD).

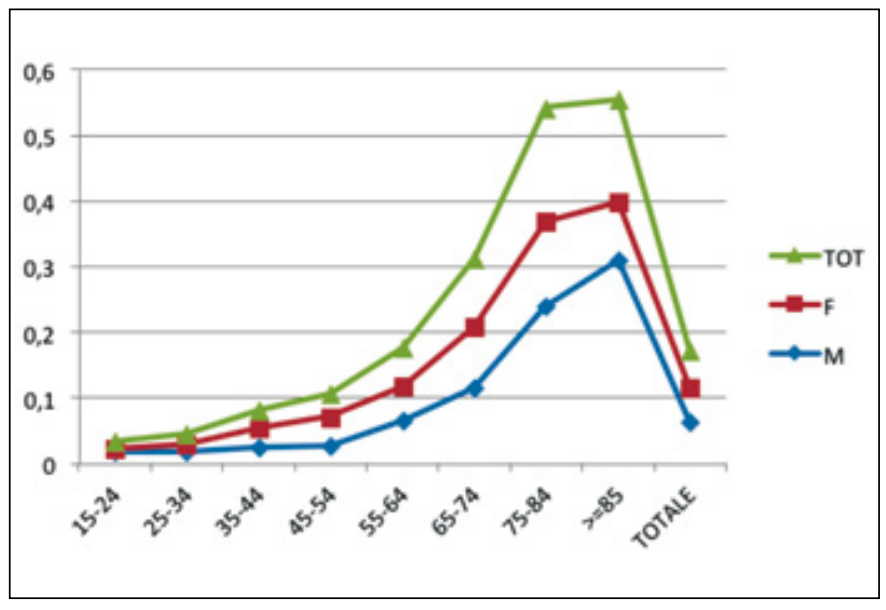

Grafico 2 - 753.1 Malattia cistica rene.

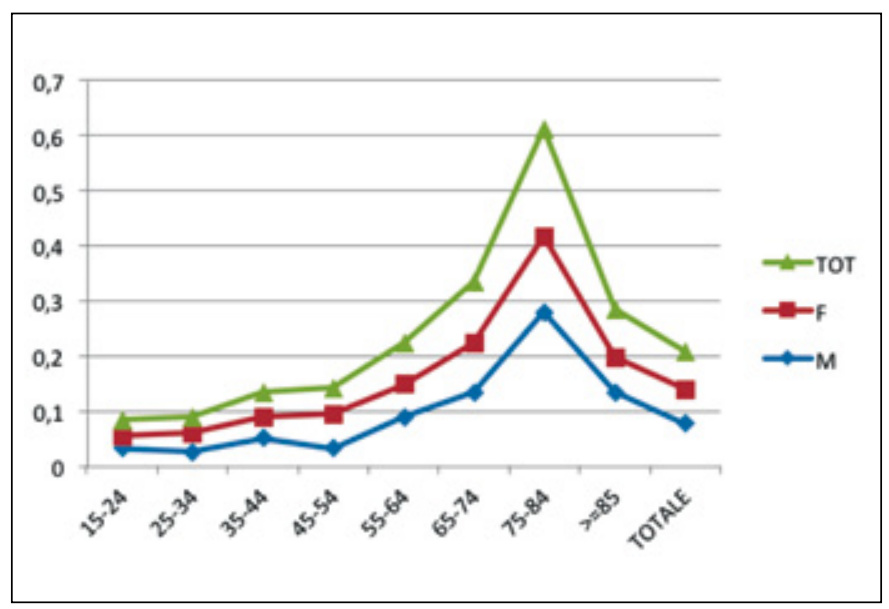

Grafico 4 - 753.12 Rene Policistico.

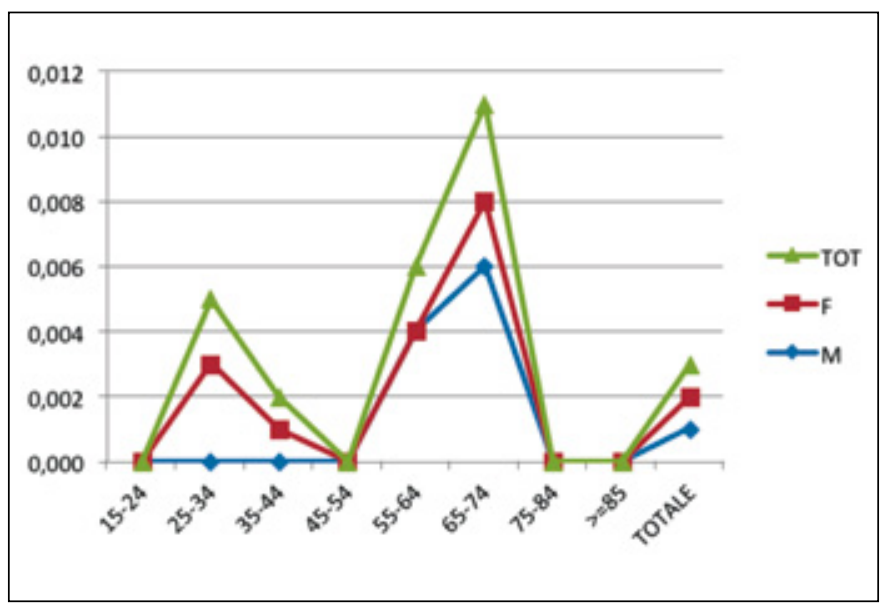

Grafico 6 - 753.14 Rene Policistico Autosomico Recessivo (ARPKD).

(c) Wichtig Editore 2012 


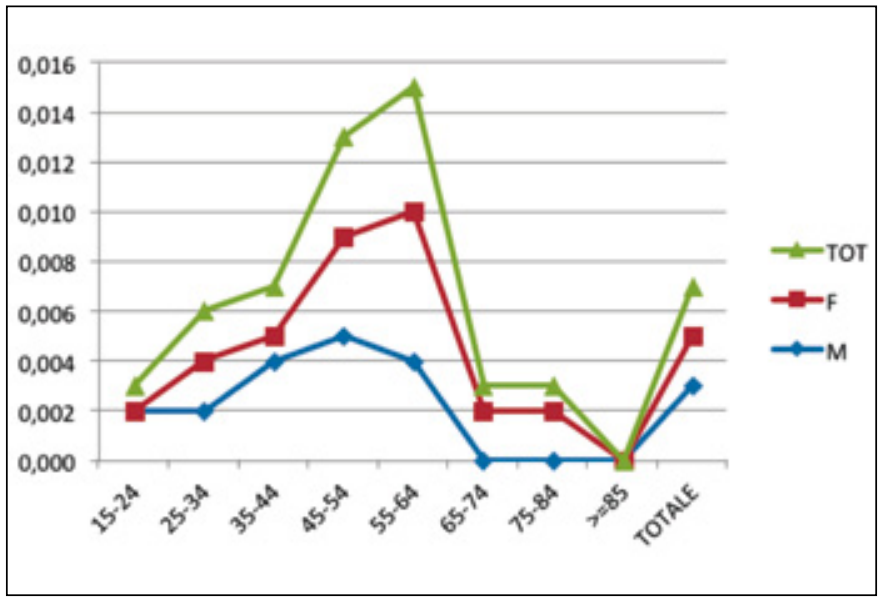

Grafico 7 - 753.17 Rene con midollare a spugna.

mo; è stata un'occasione per conoscere un'associazione di pazienti che si sta prodigando per diffondere la conoscenza della malattia e per ottenerne l'attenzione del mondo socio-sanitario. Una delle criticità di sistema che è emersa, oltre alla carente conoscenza della malattia, riguarda l'assenza di riconoscimento ai fini dell'esenzione alla partecipazione alla spesa sanitaria e che riguardi la persona affetta, indipendentemente dallo stadio di malattia renale cronica e/o dalla percentuale di invalidità riconosciuta. L'esperienza comune ha rinforzato le nostre motivazioni all'impegno che si era già manifestato con la progettazione di un primo studio preliminare, valutativo epidemiologico, sulla malattia.

\section{Studio epidemiologico preliminare sulla prevalenza dell'ADPKD in Italia}

Lo studio è stato fatto proprio dalla direzione regionale della SIMG-Sicilia, con il coinvolgimento delle diverse sezioni provinciali; ha avuto lo start-up nel marzo 2012, si è concluso nel maggio 2012 e i risultati sono stati presentati al Congresso Regionale della SIMG-Sicilia (Palermo, 18/19 maggio 2012).

Motivazioni e contesto. Le considerazioni 1, 2 e 3 esposte nelle motivazioni dell'impegno della MG per l'ADPKD hanno portato alla progettazione dello studio.

Gli obiettivi dello studio sono stati due.

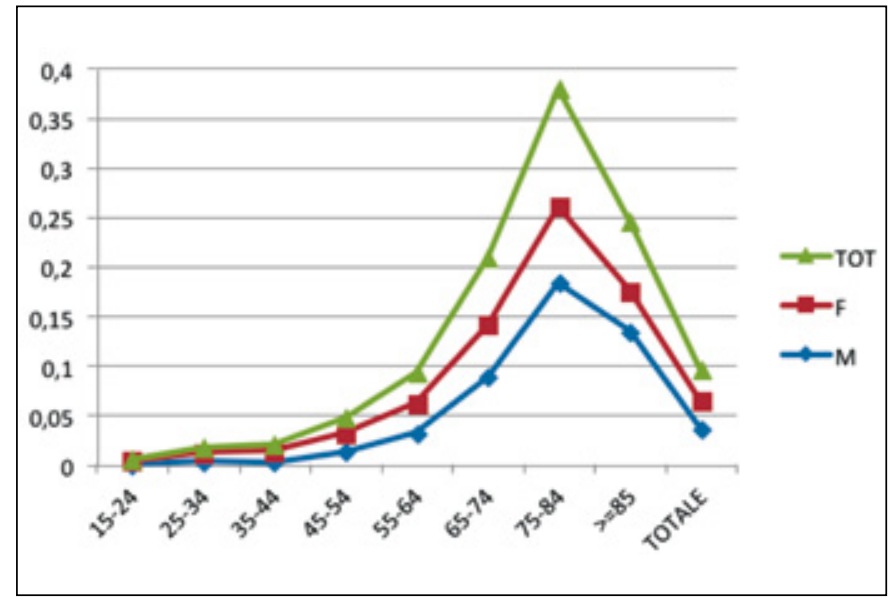

Grafico 8 - 753.19 Rene Multicistico.

Il primo ha voluto rilevare la prevalenza della ADPKD nel territorio nazionale con un'estrazione di dati da Health Search-CSD LPD, database e Istituto di ricerca della Medicina Generale Italiana, nel quale confluiscono i dati di circa 1200000 soggetti, campione statisticamente rappresentativo dell'intera popolazione italiana.

Il secondo obiettivo ha voluto verificare, in un campione di MMG della regione Sicilia, le diagnosi riconducibili alla presenza di cisti renali e tra queste selezionare quelle riferibili ad ADPKD. Questo secondo obiettivo è derivato dalla necessità di verifica su una presupposta inadeguata codifica ICD-IX, sul software di studio della MG, delle malattie cistiche renali e conseguenziale influenza sulla loro rilevanza epidemiologica. La revisione caso per caso delle condizioni comportanti cisti renali ha consentito di avere dati epidemiologici quanto più vicini al vero.

Metodi. Relativamente al primo obiettivo, nel mese di marzo 2012, è stata eseguita sul database Health Search-CSD LPD un'estrazione dati riguardanti le prevalenze, per genere e fasce d'età e relativamente al 2010, delle seguenti condizioni:

\subsection{CISTI RENALE ACQUISITA; CISTI RENE}

753.1 MALATTIA CISTICA RENE

753.11 CISTI RENALE CONGENITA, SINGOLA

753.12 RENE POLICISTICO

753.13 RENE POLICISTICO AUTOSOMICO DOMINANTE (ADPKD) 


\subsection{RENE POLICISTICO AUTOSOMICO RECESSIVO}

753.17 RENE CON MIDOLLARE A SPUGNA 753.19 RENE MULTICISTICO

I dati ottenuti sono riassunti nei grafici che seguono, in cui sull'asse delle ordinate è indicata la prevalenza (\%o) e su quella delle ascisse le classi d'età, e nella Tabella III.

La prevalenza dell'ADPKD nel database HS (1/1000) conferma quella nota dalla letteratura internazionale. L'esame della distribuzione dei casi di condizione cistica renale diversa dall'ADPKD porta a sospettare che tra quei casi possano esservi ADPKD. La possibile non perfetta conoscenza delle caratteristiche diagnostiche dell'ADPKD potrebbe aver portato a un errore di codifica e quindi a dati epidemiologici errati. Il secondo obiettivo ha voluto verificare tale ipotesi e ha previsto la selezione di 50 MMG siciliani nelle nove provincie, uno per ogni 100000 abitanti. Ciascun MMG ha ricevuto il progetto dello studio contenente anche dati informativi sulle caratteristiche dell'ADPKD e ha estratto dal proprio database di studio i pazienti che avevano la codifica per le condizioni elencate in Tabella III, riportandone i dati, divisi per genere, su una scheda. Successivamente, il medico ha rivalutato tutti i propri casi con presenza di cisti renali e tra questi ha verificato quali avessero le caratteristiche per ADPKD, sulla base dei criteri clinico-strumentali già descritti a proposito della diagnosi. Per ogni caso di verosimile $A D P K D$, il MMG ha riportato su una seconda scheda: iniziali, provincia di residenza, età anagrafica, età alla diagnosi, genere, ultima clearance della creatinina, presenza di ipertensione arteriosa, presenza di altre comorbidità (campo libero), altre localizzazioni dell'ADPKD, presenza di familiarità e l'eventuale esecuzione del test diagnostico genetico.

\section{Risultati}

I medici che hanno partecipato allo studio sono stati 40, per una popolazione assistita di 56619 unità. Nella Tabella IV sono riassunti i casi comportanti la presenza di cisti renali e ottenuti dalla estrazione automatica dai software di studio. Da questa estrazione, la prevalenza dell'ADPKD è risultata dello $0.5 \%$, con un rapporto $\mathrm{M}: \mathrm{F}$ di

TABELLA III - QUADRO SINOTTICO DELLE PREVALENZE (\%) RILEVATE PER LE CONDIZIONI INDAGATE SUL DATABASE HEALTH SEARCH-CSD LPD

\begin{tabular}{|l|c|c|c|}
\hline & M & F & 0.057 \\
\hline 593.2 CISTI RENALI ACQUISITA; CISTI RENE & 0.063 & 0.051 & 0.057 \\
\hline 753.1 MALATTIA CISTICA RENE & 0.063 & 0.051 & 0.053 \\
\hline 753.11 CISTI RENALE CONGENITA, SINGOLA & 0.055 & 0.052 & 0.069 \\
\hline 753.12 RENE POLICISTICO & 0.078 & 0.061 & $\mathbf{0 . 0 0 1}$ \\
\hline 753.13 RENE POLICISTICO AUTOSOMICO DOMINANTE (ADPKD) & $\mathbf{0 . 0 0 2}$ & $\mathbf{0 . 0 0 1}$ & 0.001 \\
\hline 753.14 RENE POLICISTICO AUTOSOMICO RECESSIVO & 0.001 & 0.001 & 0.002 \\
\hline 753.17 RENE CON MIDOLLARE A SPUGNA & 0.003 & 0.002 & 0.032 \\
\hline 753.19 RENE MULTICISTICO & 0.036 & 0.028 & \\
\hline
\end{tabular}


1.9. Tutti i casi comportanti cisti sono stati sottoposti a revisione e sono stati ricondotti a diagnosi di ADPKD altri 77 casi, portando la prevalenza finale a $1.9 \%$, quasi quadruplicata rispetto a quella ottenuta con la prima estrazione; il rapporto $\mathrm{M}: \mathrm{F}$ è stato di 1.65 . In Tabella $\mathrm{V}$ sono riassunti i dati della prima estrazione e dopo la rivalutazione di tutti i casi.

Nei 106 casi di ADPKD, l'età media anagrafica è stata di 62 anni, mentre quella media alla diagnosi di 53 anni. L’ipertensione era presente nell' $83 \%$ dei casi ed è stata descritta una comorbidità, rappresentata soprattutto da ipertensione arteriosa, cisti in altre sedi, cardiopatia ischemica, valvulopatie, aneurismi, ernie, diverticolosi del colon ecc. Nessuno dei casi risultava aver avuto una diagnosi genetica. Nel corso dello studio sono emerse difficoltà, soprattutto, relative alla non esatta conoscenza del profilo dell'ADPKD da parte dei MMG, con difficoltà

TABELLA IV - DATI OTTENUTI DALL'ESTRAZIONE AUTOMATICA DAI SOFTWARE DI STUDIO

\begin{tabular}{|l|c|c|c|c|}
\hline & M & F & Tot & \%o \\
\hline 593.2 CISTI RENALI ACQUISITA; CISTI RENE & 149 & 151 & 300 & 5.3 \\
\hline 753.1 MALATTIA CISTICA RENE & 59 & 37 & 96 & 1.8 \\
\hline 753.11 CISTI RENALE CONGENITA, SINGOLA & 38 & 23 & 61 & 1.1 \\
\hline 753.12 RENE POLICISTICO & 22 & 11 & 33 & 0.6 \\
\hline 753.13 (ADPKD) & 19 & 10 & 29 & 0.5 \\
\hline 753.14 RENE POLICISTICO AUTOSOMICO RECESSIVO & 1 & 1 & 2 & 0.04 \\
\hline 753.17 RENE A SPUGNA & 2 & 0 & 2 & 0.04 \\
\hline 753.19 RENE MULTICISTICO & 25 & 14 & 39 & 0.7 \\
\hline
\end{tabular}

TABELLA V - DATI DELLA PRIMA ESTRAZIONE E DOPO LA RIVALUTAZIONE DI TUTTI I CASI

\begin{tabular}{|l|c|c|c|c|c|}
\hline & M & F & Tot & \%o & M:F \\
\hline $\begin{array}{l}753.13 \text { (ADPKD) } \\
\text { Prima della verifica }\end{array}$ & 19 & 10 & 29 & 0.5 & 1.9 \\
\hline Nuovi casi dopo la verifica & 47 & 30 & 77 & & \\
\hline 753.13 (ADPKD) & 66 & 40 & $\underline{\mathbf{1 0 6}}$ & $\underline{\mathbf{1 . 9}}$ & $\underline{\mathbf{1 . 6 5}}$ \\
\hline
\end{tabular}

TABELLA VI - CARATTERISTICHE DELLA POPOLAZIONE ADPKD

\begin{tabular}{|c|c|c|c|c|c|}
\hline \multicolumn{2}{|c|}{ Età } & \multirow{2}{*}{$\begin{array}{l}\text { Ipert. } \\
\text { Arter. }\end{array}$} & $\begin{array}{c}\text { Altre } \\
\text { comorbidità }\end{array}$ & $\begin{array}{c}\text { Familiari } \\
\text { ADPKD }\end{array}$ & $\begin{array}{c}\text { Diagnosi } \\
\text { genetica }\end{array}$ \\
\hline Anagr. & Alla dia. & & & & Nessuna \\
\hline 62 & 53 & $88(83 \%)$ & $80 \%$ & 20 & $(20 \%)$ \\
\hline
\end{tabular}


a identificare i casi, la precedente non avvenuta codifica delle "cisti renali" tra i problemi aperti nella scheda-paziente e l'annessione di referti ecografici sotto altre diagnosi (Ipertensione, IRC ecc.). È emersa la carente conoscenza delle caratteristiche sistemiche della malattia.

\section{Conclusioni}

L'ADPKD, pur essendo la causa di terapia sostitutiva emodialitica nel 5-10\% dei pazienti, con inferenze anche sui familiari come malattia genetica autosomica dominante, è una condizione poco conosciuta presso la MG nel suo corretto profilo diagnostico e clinico. L'ipotesi che la sua diagnosi potesse essere confusa con altre condizioni che comportano la presenza di cisti nei reni è stata confermata da uno studio preliminare di prevalenza nel setting della medicina generale, per cui è verosimile che la prevalenza sia superiore rispetto a quella valutata di 1:1000. E auspicabile l'impegno della MG per la diffusione della conoscenza della condizione contribuendo e collaborando per gli oneri e gli impegni socioscientifici già assunti dalle Associazioni dei pazienti e dalle Società Scientifiche nefrologiche.

\section{Riassunto}

La malattia policistica renale autosomica dominante dell'adulto (ADPKD) è una delle malattie genetiche più comuni, con un'incidenza di $1 \mathrm{su} 1000$, ed è la principale causa genetica di insufficienza renale dell'adulto. Se ne distinguono due tipi; il tipo I, causato da mutazioni del gene PKD1, e il tipo II, causato da mutazioni del gene PKD2. La malattia ha un esordio tra i 40 e i 50 anni, ma può manifestarsi anche prima, e ha un'impronta sistemica. La diagnosi è spesso casuale, nel corso di indagini motivate da altri quesiti clinici, oppure promossa dalla presenza di segni clinici correlati. Nelle procedure diagnostiche, l'ADPKD deve essere distinta dalle cisti renali semplici, uniche o multiple, che interessano parzialmente $\mathrm{i}$ reni e che generalmente non presentano un'evoluzione critica. È possibile che nell'attività clinica possano essere diagnosticate erroneamente le condizioni che comportano la presenza di cisti renali, rischiando di sottovalutare il rilievo clinico dell'ADPKD. Per verificare tale ipotesi, è stato condotto uno studio epidemiologico nel setting della Medicina Generale italiana e da cui è emersa una prevalenza $(1.9 \%)$ quasi quattro volte superiore rispetto a quella registrata $(0.5 \% 0)$ prima della informazione fornita al medico di medicina generale sulle caratteristiche cliniche e diagnostiche dell'ADPKD. È auspicabile l'impegno della Medicina Generale, assieme alle Associazioni dei Pazienti a alle Società Scientifiche nefrologiche, al fine di contribuire a una ottimale gestione clinica della condizione, finalizzata al benessere ottimale del paziente.

Parole Chiave. ADPKD, Rene policistico autosomico dominante, Medicina generale, Insufficienza renale, Dialisi

Indirizzo degli Autori:

Dr. Salvatore Campo

Via Bellini 16/a

90041 Balestrate (PA)

campo.salva@tin.it

\section{Bibliografia}

1. Igarashi P, Somlo S. Genetics and pathogenesis of polycystic kidney disease. J Am Soc Nephrol 2002; 13: 2384-98.

2. Hateboer N, v Dijk MA, Bogdanova N, et al. Com- parison of phenotypes of polycystic kidney disease types 1 and 2. European PKD1-PKD2 Study Group. Lancet 1999; 353: 103-07.

3. Parfrey PS, Bear JC, Morgan J, et al. The diagnosis and prognosis of autosomal dominant polycystic kidney disease. N Engl J Med 1990; 323: 1085-90.

4. Ravine D, Walker RG, Gibson RN, et al. Phenot- 
ype and genotype heterogeneity in autosomal dominant polycystic kidney disease. Lancet 1992; 340: 1330-3.

5. Pei Y, Paterson AD, Wang KR, et al. Bilineal disease and trans-heterozygotes in autosomal dominant polycystic kidney disease. Am J Hum Genet 2001; 68: 355- 63.

6. Rossetti S, Consugar M, Chapman A, et al, CRISP Consortium. Comprehensive molecular diagnostics in autosomal dominant polycystic kidney disease. J Am Soc Nephrol 2007; 18: 2143-60.

7. Garcia-Gonzalez M, Jones J, Allen S, et al. Evaluating the clinical utility of a molecular genetic test for polycystic kidney disease. Mol Genet Metab 2007; 92: 160-7.

8. Ravine D, Gibson RN, Walker RG, Sheffield L,
Kincaid-Smith P, Danks D. Evaluation of ultrasonographic diagnostic criteria for autosomal dominant polycystic kidney disease 1 . Lancet 1994; 343: 824-7.

9. Pei Y, Obaji J, Dupuis A, et al. Unified Criteria for Ultrasonographic Diagnosis of ADPKD. J Am Soc Nephrol (JASN) 2009; 20(1): 205-12.

10. Shillingford JM, Murcia NS, Larson $\mathrm{CH}$, et al. The mTOR pathway is regulated by polycystin-1 and its inhibition reverses renal cystogenesis in polycystic kidney disease. Proceedings of the National Academy of Sciences of the USA 2006; 103: 5466-71.

11. Grantham JJ, Bennett WM, Perrone RD. mTOR Inhibitors and Autosomal Dominant Polycystic Kidney Disease. N Engl J Med 2011; 364: 286-9. 